\title{
Classifier Based Low-Complexity MIMO Detection for Spatial Multiplexing Systems
}

\author{
C. R. N. Athaudage †, M. Zhang*, A. D. S. Jayalath††, and T. D. Abhayapala* \\ $\dagger$ ARC Special Research Center for Ultra-Broadband Information Networks (CUBIN) \\ Department of Electrical and Electronic Engineering \\ University of Melbourne, Australia \\ * Research School of Information Engineering \\ Australian National University, Canberra, Australia \\ †† School of Engineering Systems \\ Queensland University of Technology, Brisbane, Australia
}

\begin{abstract}
In this paper, we propose a low complexity detection scheme for MIMO systems incorporating spatial multiplexing. Optimal detection schemes such as maximum-likelihood (ML) detection of MIMO signals demands computational resources that are beyond the capabilities of most practical systems. Alternative reduced complexity MIMO detection techniques have been proposed, but the complexity of algorithmic schemes are in general much higher than that of the equalizer-based techniques, e.g. zero-forcing (ZF) or MMSE. On the other hand, equalizerbased techniques perform relatively poor in terms of error rate. In this paper, we propose a hybrid of an equalizer-based technique and an algorithmic search stage. Based on an error matric and its probability density functions for different classes of error, a particular search region is selected for the algorithmic stage. As the probability of occurrence of error classes with larger search regions is small, overall complexity of the proposed technique remains low while providing a significant improvement in the error performance.
\end{abstract}

\section{INTRODUCTION}

Multiple-input multiple-output (MIMO) systems offer both high data rates and high link reliability for mobile wireless communications due to the inherent space diversity. Spatial multiplexing (SM) MIMO systems provide higher data rates as each transmit antenna emits an independent information symbol in different time slots, i.e with $N_{t}$ transmit antennas, the data rate can be increased by a factor of $N_{t}$. On the other hand, the achievable diversity order (negative exponent of the error rate at high SNR) in SM-MIMO depends on the detection (or equalization) technique incorporated at the MIMO receiver. The optimal detector that minimizes the average error probability is the maximum-likelihood (ML) detector [1]. However, the ML-detector practically infeasible as its computational complexity is exponential. Different algorithms generally known as sphere decoders have been developed to achieve near ML performance with polynomial complexity [2]. On the other end of the complexity spectrum the equalizer based MIMO detection schemes can be found. These include zero-forcing (ZF) detector [3] and minimum-mean-squareerror (MMSE) detector [4]. The ZF-detector and the MMSEdetector have the minimal computational burden as they require only matrix operations, e.g. pseudo-inverse. However, the error performance of both ZF and MMSE detectors are significantly lower than the optimal ML detector. Note that both ZF-detector and MMSE-detector have a diversity order of $N_{r}-N_{t}+1$, while the optimal ML-detector has a diversity order of $N_{r}$, where $N_{r}$ is the number of receive antennas. The successive interference cancelation schemes such as VBLAST for MIMO detection achieves only a fraction of the diversity available in the MIMO channel. Also the iterative nature of VBLAST adds to its computational complexity.

Even though the integrated-circuit technology is available today to implement the most complex MIMO detection schemes such as ML-detector (using sphere decoding) in a silicon chip, the actual integration cost of these detectors is out of scope for most of the expected applications of MIMO systems. Thus the quest is still on to find a MIMOdetector with a near ML error performance while having a low complexity close to ZF-detector.

In this context, we propose a classification-based detector for SM-MIMO systems as a good compromise between the error performance and complexity. The proposed detector starts with a equalizer-based (ZF or MMSE) symbol estimate and then based on an error measure a search region is selected. Finally within the selected search region an ML-type search is performed to find out the error minimizing symbol vector.

This paper is organized as follows. Section II introduces the MIMO signal model used. The details the proposed classifier based MIMO detector are given in Section III. Simulation results and performance comparisons are presented in Section IV. The concluding remarks are given in Section V.

\section{SM-MIMO Signal ModeL}

A spatial multiplexed MIMO system with $N_{t}$ number of transmit antennas and $N_{r}$ number of receive antennas is considered. The transmitted symbol vector for a given time slot is taken as $\mathbf{x}=\left[\begin{array}{llll}x_{0} & x_{1} & \ldots & x_{N_{t}-1}\end{array}\right]^{T}$, with $\sigma_{x}^{2}=$ $E\left\{\left|x_{n}\right|^{2}\right\}$ for $n=0,1, \ldots, N_{t}-1$ is the signal power. The received signal vector $\mathbf{r}=\left[\begin{array}{llll}r_{0} & r_{1} & \ldots & r_{N_{r}-1}\end{array}\right]^{T}$ corresponding to $\mathrm{x}$ can be given as

$$
\mathbf{r}=\mathbf{H} \mathbf{x}+\mathbf{w}
$$


where, $\mathbf{H}$ is the $N_{r} \times N_{t}$ channel matrix containing statistically independent complex Gaussian channel values. The channel noise (AWGN) vector is given by $\mathbf{w}=$ $\left[\begin{array}{llll}w_{0} & w_{1} & \ldots & w_{N_{r}-1}\end{array}\right]^{T}$ with $\sigma_{w}^{2}=E\left\{\left|w_{m}\right|^{2}\right\}$ for $m=$ $0,1, \ldots, N_{r}-1$ is the noise power. The element $h_{i j}$ corresponding to the $i$ th row and the $j$ th column of the matrix $\mathbf{H}$ is a zero-mean complex Gaussian random variable with unit power, i.e. $\Re\left\{h_{i j}\right\} \sim \mathcal{N}(0,1 / 2)$ and $\Im\left\{h_{i j}\right\} \sim \mathcal{N}(0,1 / 2)$, where $\Re\{$.$\} and \Im\{$.$\} denotes the real and imaginary parts$ of a complex number, respectively. A real Gaussian random variable with mean $\mu$ and variance $\sigma^{2}$ is given by $\mathcal{N}\left(\mu, \sigma^{2}\right)$. The equalizer-based MIMO detection can be generalized as

$$
\mathbf{x}_{\mathrm{EQ}}=\mathbf{G r}
$$

where, $\mathbf{G}$ is the $N_{t} \times N_{r}$ equalizer matrix. The estimated transmitted symbol vector $\hat{\mathbf{x}}$ becomes

$$
\hat{\mathbf{x}}_{\mathrm{EQ}}=Q\left(\mathbf{x}_{\mathrm{EQ}}\right)
$$

where, $Q($.$) denotes the nearest neighbor constellation demap-$ ping operation. For ZF-equalization $\mathbf{G}=\mathbf{G}_{\mathrm{ZF}}$ is [3]

$$
\mathbf{G}_{\mathrm{ZF}}=\left(\mathbf{H}^{\mathcal{H}} \mathbf{H}\right)^{-1} \mathbf{H}^{\mathcal{H}}
$$

where, (.) $)^{\mathcal{H}}$ is the conjugate transpose of a matrix. Also, $\mathbf{H}^{\dagger}=$ $\left(\mathbf{H}^{\mathcal{H}} \mathbf{H}\right)^{-1} \mathbf{H}^{\mathcal{H}}$ is known as the pseudo-inverse of the matrix $\mathbf{H}$. For MMSE-equalization $\mathbf{G}=\mathbf{G}_{\mathrm{MMSE}}$ is [4]

$$
\mathbf{G}_{\text {MMSE }}=\left(\mathbf{H}^{\mathcal{H}} \mathbf{H}+\frac{\sigma_{w}^{2}}{\sigma_{x}^{2}} \mathbf{I}\right)^{-1} \mathbf{H}^{\mathcal{H}} .
$$

The optimal ML-detector can be given as [1]

$$
\hat{\mathbf{x}}_{\mathrm{ML}}=\arg \min _{\tilde{\mathbf{x}} \in \mathcal{D}}\|\mathbf{r}-\mathbf{H} \tilde{\mathbf{x}}\|^{2}
$$

where, $\mathcal{D}$ denotes the set of all possible transmitted symbol vectors. For an $M$-ary constellation $|\mathcal{D}|=M^{N_{t}}$.

\section{Proposed Classification Based Detector}

The proposed detector starts with a equalizer based estimate $\hat{\mathbf{x}}_{\mathrm{EQ}}$ of the transmitted symbol vector. Thus $\hat{\mathbf{x}}_{\mathrm{EQ}}=\hat{\mathbf{x}}_{\mathrm{ZF}}$ or $\hat{\mathbf{x}}_{\mathrm{EQ}}=\hat{\mathbf{x}}_{\mathrm{MMSE}}$. We define the symbol error vector as

$$
\mathbf{e}=\mathbf{x}-\hat{\mathbf{x}}_{\mathrm{EQ}}
$$

For the $M$-ary constellation of $\mathcal{C}=\left\{c_{0}, c_{1}, \ldots, c_{M-1}\right\}$, it should be noted that $\mathbf{x}, \hat{\mathbf{x}}_{\mathrm{EQ}} \in \mathcal{C}^{N_{t}}$ and $\mathbf{e} \in \mathcal{E}^{N_{t}}$, where $\mathcal{E}=$ $\left\{c_{p}-c_{q} \mid p, q=0,1, \ldots, M\right\}$. We define the error measure $\rho(\hat{\mathbf{x}})$ w.r.t. to any given symbol vector estimate $\hat{\mathbf{x}}$ as

$$
\rho(\hat{\mathbf{x}})=\|\mathbf{r}-\mathbf{H} \hat{\mathbf{x}}\|^{2} .
$$

If $\hat{\mathbf{x}}=\mathbf{x}$, i.e. perfect estimate, then from (1) and (8) $\rho(\hat{\mathbf{x}})$ becomes

$$
\rho(\mathbf{x})=\|\mathbf{w}\|^{2}=\sum_{m=0}^{N_{r}-1}\left|w_{m}\right|^{2}
$$

From (9) it is clear that $\rho(\mathbf{x})$ is a chi-square random variable with degree $2 N_{r}$.

Remark 1: The notation $\chi_{n}^{2}\left(\sigma^{2}\right)$ is used for a Chi-square (or Gamma) random variable with $n$-degree of freedom and has the form $Y=\sum_{i=1}^{n} X_{i}^{2}$, where $X_{i} \sim \mathcal{N}\left(0, \sigma^{2}\right)$ are real and independent. The PDF of $Y$ is given by [5] (pp. 41-42)

$$
p_{y}(y)=\frac{1}{\sigma^{n} 2^{n / 2} \Gamma(n / 2)} y^{n / 2-1} e^{-y / 2 \sigma^{2}}
$$

where, the Gamma function $\Gamma(p)=(p-1)$ ! for integer $p>0$. Thus $\rho(\mathbf{x}) \sim \chi_{2 N_{r}}^{2}\left(\sigma_{w}^{2} / 2\right)$.

Lets define a class of estimates $\hat{\mathbf{x}} \in \mathcal{G}_{1}$ as an estimate which is different from $\mathbf{x}$ only by one symbol and this difference is an adjacent constellation point, i.e. the symbol error vector $\mathbf{e}=\mathbf{x}-\hat{\mathbf{x}}=\left[\begin{array}{llll}e_{0} & e_{1} & \ldots & e_{N_{t}-1}\end{array}\right]^{T}$ has the form $e_{n}=0$ for all $n$ except for $n=n_{1}$ and $e_{n_{1}}=\Delta_{1} u_{1}$, where $u_{1}$ is unitmagnitude $\left(\left|u_{1}\right|=1\right)$ complex number and $\Delta_{1}$ is the distance between two adjacent constellation points (assume an $M$-PSK constellation - see Fig. 1). Thus the error measure $\rho\left(\hat{\mathbf{x}} \in \mathcal{G}_{1}\right)$ becomes

$$
\begin{aligned}
\rho\left(\hat{\mathbf{x}} \in \mathcal{G}_{1}\right) & =\|\mathbf{r}-\mathbf{H} \hat{\mathbf{x}}\|^{2} \\
& =\|\mathbf{w}+\mathbf{H e}\|^{2} \\
& =\sum_{m=0}^{N_{r}-1}\left|w_{m}-\Delta_{1} u_{1} h_{m n_{1}}\right|^{2}
\end{aligned}
$$

Note that $\xi_{m}=w_{m}-\Delta_{1} u_{1} h_{m n_{1}}$ in (11) is a complex Gaussian variable with zero mean and variance $\sigma_{\xi}^{2}=\sigma_{w}^{2}+\left|\Delta_{1}\right|^{2} \sigma_{h}^{2}$ (We have treated $h_{m n_{1}}$ as a random variable here such that the classifier design becomes only dependent on the channel statistics.). Thus we have

$$
\rho\left(\hat{\mathbf{x}} \in \mathcal{G}_{1}\right) \sim \chi_{2 N_{r}}^{2}\left(\left(\sigma_{w}^{2}+\left|\Delta_{1}\right|^{2} \sigma_{h}^{2}\right) / 2\right) .
$$

Similarly, we can define a class of estimates $\hat{\mathbf{x}} \in \mathcal{G}_{2}$ as an estimate which is different from $\mathbf{x}$ only by two symbols and these differences are by an adjacent constellation point, i.e. the symbol error vector $\mathbf{e}=\mathbf{x}-\hat{\mathbf{x}}=\left[\begin{array}{llll}e_{0} & e_{1} & \ldots & e_{N_{t}-1}\end{array}\right]^{T}$ has the form $e_{n}=0$ for all $n$ except for $n=n_{1}$ and $n=n_{2}$ with $e_{n_{1}}=\Delta_{1} u_{1}$ and $e_{n_{2}}=\Delta_{1} u_{2}$, where $u_{1}$ and $u_{2}$ are unit-magnitude $\left(\left|u_{1}\right|=\left|u_{2}\right|=1\right)$ complex numbers. Similar to the error class $\mathcal{G}_{1}$, it can be shown that

$$
\rho\left(\hat{\mathbf{x}} \in \mathcal{G}_{2}\right) \sim \chi_{2 N_{r}}^{2}\left(\left(\sigma_{w}^{2}+2\left|\Delta_{1}\right|^{2} \sigma_{h}^{2}\right) / 2\right) .
$$

Also, we can define a class of estimates $\hat{\mathbf{x}} \in \mathcal{G}_{3}$ as an estimate which is different from $\mathbf{x}$ only by one symbol and this difference is by two adjacent constellation point 2 , i.e. the symbol error vector $\mathbf{e}=\mathbf{x}-\hat{\mathbf{x}}=\left[\begin{array}{llll}e_{0} & e_{1} & \ldots & e_{N_{t}-1}\end{array}\right]^{T}$ has the form $e_{n}=0$ for all $n$ except for $n=n_{3}$ and $e_{n_{3}}=\Delta_{2} u_{3}$, where $u_{3}$ is unit-magnitude $\left(\left|u_{3}\right|=1\right)$ complex number and $\Delta_{2}$ is the distance between two constellation points separated by only one constellation point (see Fig. 1). And we have

$$
\rho\left(\hat{\mathbf{x}} \in \mathcal{G}_{3}\right) \sim \chi_{2 N_{r}}^{2}\left(\left(\sigma_{w}^{2}+\left|\Delta_{2}\right|^{2} \sigma_{h}^{2}\right) / 2\right) .
$$

In this fashion, we can generate the total possible set of error classes $\mathcal{G}_{0}, \mathcal{G}_{1}, \ldots, \mathcal{G}_{G}$ with the error measure $\rho\left(\hat{\mathbf{x}} \in \mathcal{G}_{g}\right)$ for $g=0,1, \ldots, G$ having a statistical distribution in the increasing order of the variance of $\chi_{2 N_{r}}^{2}$ (.).

Remark 2: The exact probability density function (PDF) $p_{\mathcal{G}_{g}}\left(\rho \mid \mathcal{G}_{g}\right)$ of $\rho\left(\hat{\mathbf{x}} \in \mathcal{G}_{g}\right)$ can be calculated using (10) with 


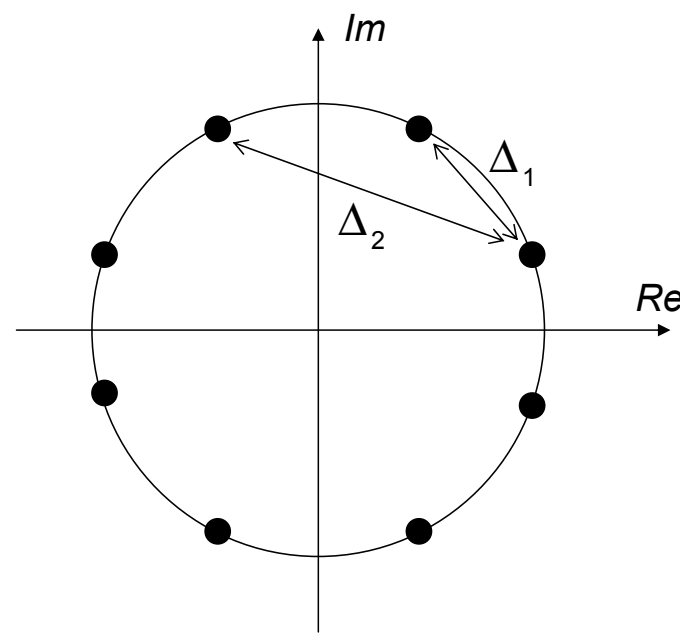

Fig. 1. Adjacent constellation point distances $\Delta_{1}$ and $\Delta_{2}$ for 8-PSK.

the substitutions $y=\rho, n=2 N_{r}$, and the corresponding variance $\sigma^{2}$ for the class $\mathcal{G}_{g}$, i.e. for $\mathcal{G}_{1}, \sigma^{2}=\sigma_{w}^{2} / 2$ and for $\mathcal{G}_{2}, \sigma^{2}=\left(\sigma_{w}^{2}+\left|\Delta_{1}\right|^{2} \sigma_{h}^{2}\right) / 2$ and so forth.

Remark 3: The total number of error classes $G$ and the sizes of the individual classes $\left|\mathcal{G}_{g}\right|$ for $g=0,1, \ldots, G$ depend on the number of transmit antennas $N_{t}$ and the constellation size $M$. For an example $\left|\mathcal{G}_{0}\right|=1$ and, $\left|\mathcal{G}_{1}\right|=2 N_{t}$. Also for BPSK $(M=2), G=N_{t}+1$.

Remark 4: The universal set $\mathcal{G}=\mathcal{G}_{0} \cup \mathcal{G}_{1} \cup \ldots \cup \mathcal{G}_{G}$ contains all possible symbol vectors of size $N_{t}$, thus $|\mathcal{G}|=M^{N_{t}}$. Also $\mathcal{G}$ is equal to $\mathcal{C}$ in (6), i.e. the search space for $\mathrm{ML}$ detection. Therefore, the proposed classification based detector divides the total ML search space into $G$ number of mutually exhaustive and exclusive subspaces.

\section{A. Proposed Algorithm}

Based on the above classification the proposed MIMO detection algorithm can be formulated as follows:

Step 1: Calculate the error measure $\rho$ using an equalizer-based estimate of the transmitted symbol vector $\hat{\mathbf{x}}_{\mathrm{EQ}}$ (either $\mathrm{ZF}$ or MMSE):

$$
\rho=\left\|\mathbf{r}-\mathbf{H} \hat{\mathbf{x}}_{\mathrm{EQ}}\right\|^{2} .
$$

Step 2: Given the observation $\rho_{\mathrm{EQ}}$, calculate according to the Bayesian classification rule the most probable class index $i^{*}$ that $\hat{\mathbf{x}}_{\mathrm{EQ}}$ belongs to:

$$
i^{*}=\arg \max _{i} P_{\mathcal{G}_{i}}\left(\mathcal{G}_{i} \mid \rho\right)
$$

where, $P_{\mathcal{G}_{i}}\left(\mathcal{G}_{i} \mid \rho\right)$ is the probability that for a given $\rho$ it comes from the class $\mathcal{G}_{i}$. But

$$
P_{\mathcal{G}_{i}}\left(\mathcal{G}_{i} \mid \rho\right)=\frac{p_{\mathcal{G}_{i}}\left(\rho \mid \mathcal{G}_{i}\right) P_{\mathcal{G}_{i}}}{p_{\rho}(\rho)} .
$$

where, $P_{\mathcal{G}_{i}}$ is the actual probability of occurrence of the class $\mathcal{G}_{i}$, independent of the classifier and depends on the linear equalizer used and the operating SNR. The overall PDF of $\rho$ (over all the classes) is given by $p_{\rho}(\rho)$. Thus

$$
i^{*}=\arg \max _{i} p_{\mathcal{G}_{i}}\left(\rho \mid \mathcal{G}_{i}\right) P_{\mathcal{G}_{i}} .
$$

Step 3: Perform a search within the class $\mathcal{G}_{i^{*}}$ to find out the best estimate $\hat{\mathbf{x}}^{*}$ of the transmitted symbol vector:

$$
\hat{\mathbf{x}}^{*}=\arg \min _{\tilde{\mathbf{x}} \in \mathcal{G}_{i^{*}}}\|\mathbf{r}-\mathbf{H} \tilde{\mathbf{x}}\|^{2} .
$$

In step 2 of the above algorithm higher order error classes can be ignored as their probabilities of occurrence tend to be negligible, except for very low channel SNR values. A block schematic of the proposed detector is shown in Fig 2.

\section{B. Computational Complexity}

The computational complexity of the proposed MIMO detector mainly consists of two parts: (i) complexity of obtaining the initial estimation using ZF or MMSE equalizer (Step 1). Lets denote this complexity by $\mathcal{C}_{\mathrm{EQ}}$, and (ii) complexity of performing the ML-type search within the selected subspace $\mathcal{G}_{g^{*}}$ (Step 3). This complexity can be denotes as $\beta \mathcal{C}_{\mathrm{ML}}$, where $\mathcal{C}_{\mathrm{ML}}$ is the complexity of total ML search and $\beta$ is on average the fraction of subspace searched by the proposed algorithm. The average fractional subspace can be given as

$$
\beta=\sum_{i=0}^{G} \tilde{P}_{\mathcal{G}_{i}}\left|\mathcal{G}_{i}\right|
$$

where, $\tilde{P}_{\mathcal{G}_{i}}$ is the post-classification probability of occurrence of the class $\mathcal{G}_{g}$, i.e. the probability of occurrence of the classes according the classifiers class assignment. It should be noted that $\tilde{P}_{\mathcal{G}_{i}}$ can be different from $P_{\mathcal{G}_{i}}$ due the misclassifications, i.e. inaccurate classification decisions. It is difficult to evaluate the probabilities $\tilde{P}_{\mathcal{G}_{g}}$ 's analytically, thus evaluated using simulations. The complexity associated with Step 2 can be ignored as the receiver can use a pre-calculated decision boundary based classifier rather than evaluating the PDF's of classes in real-time. The complexity $\mathcal{C}_{\mathrm{CL}}$ of the proposed classification based MIMO detector can be expressed as

$$
\mathcal{C}_{\mathrm{CL}}=\mathcal{C}_{\mathrm{EQ}}+\beta \mathcal{C}_{\mathrm{ML}}
$$

It is desirable to have $\beta$ as small as possible to assure a minimum complexity for the proposed algorithm. Also, a desirable performance versus complexity tradeoff can be achieved with the proposed algorithm by using an appropriate strategy to reduce the total number of classes $G$, e.g. ignore the classes with minimum probability of occurrence or the classes contributing minimally to the average error performance improvement. We can assure that

$$
\mathcal{C}_{\mathrm{EQ}}<\mathcal{C}_{\mathrm{CL}}<<\mathcal{C}_{\mathrm{ML}}
$$

i.e. $\mathcal{C}_{\mathrm{CL}}$ is greater than $\mathcal{C}_{\mathrm{EQ}}$ but much less than $\mathcal{C}_{\mathrm{ML}}$.

\section{Simulation Results}

In this section we provide simulation results to justify performance of the proposed classifier based MIMO detector. Performance comparisons with few other MIMO detectors are also provided. 


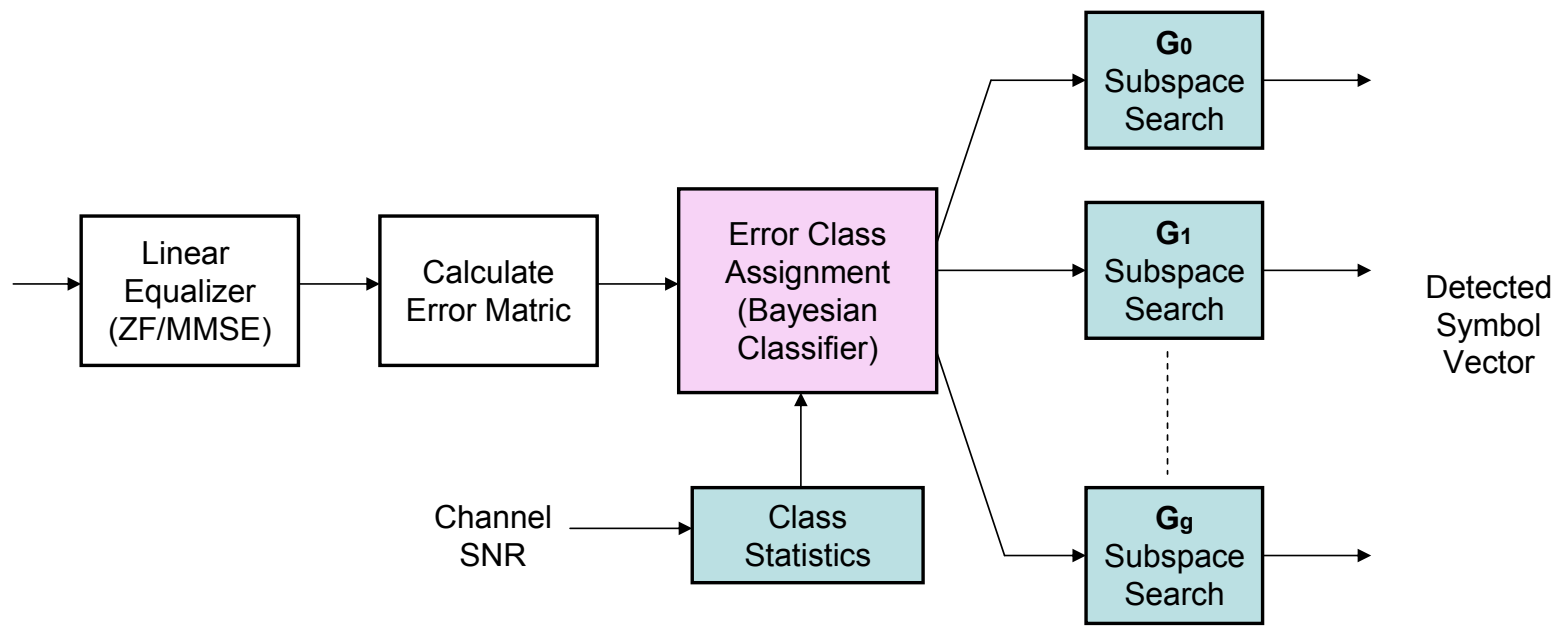

Fig. 2. Block schematic of the proposed classification based MIMO detector.

\section{A. MIMO System}

We consider a $4 \times 4 \mathrm{MIMO}$ system, i.e. $N_{t}=N_{r}=4$ with spatial multiplexing to demonstrate the performance of the proposed detection algorithm. The BPSK modulation, i.e. $M=2$, is assumed across all antennas, thus $x_{n} \in\{1,-1\}$ for $n=0,1, \ldots, N_{t}-1$, and $\Delta_{1}=2$. Also, with BPSK only adjacent constellation point errors are possible. Thus we can define 5 error classes $\mathcal{G}_{g}$, for $g=0,1, \ldots, 4$, with the class $\mathcal{G}_{g}$ meaning errors in $g$ number of locations in the estimated transmitted BPSK symbol vector. The sizes of the individual classes become $\left|\mathcal{G}_{0}\right|=1,\left|\mathcal{G}_{1}\right|=4,\left|\mathcal{G}_{2}\right|=6,\left|\mathcal{G}_{3}\right|=4$, and $\left|\mathcal{G}_{4}\right|=1$. Also, $\sum_{g=0}^{4}\left|\mathcal{G}_{g}\right|=16=2^{4}$ the size of the ML search space. The probability density function $p_{\mathcal{G}_{g}}\left(\rho \mid \mathcal{G}_{g}\right)$ of the error measure $\rho\left(\hat{\mathbf{x}} \in \mathcal{G}_{g}\right)$ becomes

$$
\rho\left(\hat{\mathbf{x}} \in \mathcal{G}_{g}\right) \sim \chi_{8}^{2}\left(\left(\sigma_{w}^{2}+g\left|\Delta_{1}\right|^{2} \sigma_{h}^{2}\right) / 2\right) \quad \forall g .
$$

\section{B. Performance Comparisons}

Figure 3 shows $p_{\mathcal{G}_{g}}\left(\rho \mid \mathcal{G}_{g}\right)$ for different classes, $g=1,2$, 3 , and 4. As can be seen, both the mean and variance of the error measure $\rho\left(\hat{\mathbf{x}} \in \mathcal{G}_{g}\right)$ increase for large error classes, i.e. as $g$ increases.

The probability of occurrence of different classes $P_{\mathcal{G}_{i}}$ against SNR for the ZF equalizer is shown in Fig. 4. As can be seen, probability of class $\mathcal{G}_{0}$ increases with SNR, while that of the other classes $\left(\mathcal{G}_{1}\right.$ to $\left.\mathcal{G}_{4}\right)$ decreases with SNR. At high SNR, e.g. SNR $>20 \mathrm{~dB}$, the class $\mathcal{G}_{0}$ dominates with more than $95 \%$ probability of occurrence. It should be noted that as SNR $\rightarrow \infty, P_{\mathcal{G}_{0}} \rightarrow 1$ and the probability of all other higher error classes goes to zero. The idea of the proposed MIMO detection scheme is to capture these error classes, thus reducing the detection complexity by minimizing the search space.

The bit-error-rate (BER) performance of the proposed classifier based detector is shown in Fig. 5. In the same figure, the performance of the following three MIMO detectors are shown for comparison: (1) ZF detector, (2) ML detector, and (3) combined ZF-ML detector [3]. As can be seen in Fig. 5,

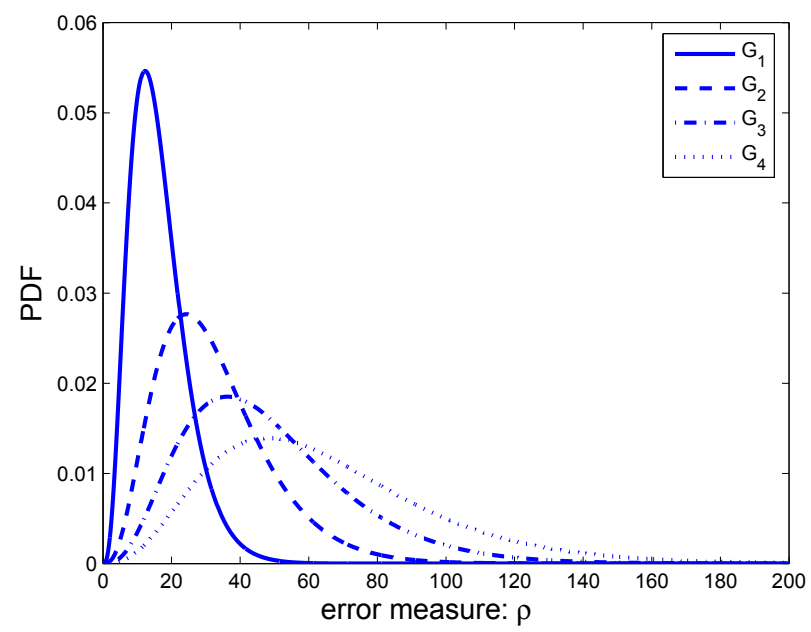

Fig. 3. Density functions of the classes $\mathcal{G}_{1}, \mathcal{G}_{2}, \mathcal{G}_{3}$, and $\mathcal{G}_{4}$ for $N_{t}=N_{r}=4$, SNR $=10 \mathrm{~dB}$, and BPSK modulation.

the BER performance of the proposed classifier based MIMO detector is significantly better than that of the ZF-detector, i.e. at $\mathrm{BER}=10^{-2}$ a $6 \mathrm{~dB}$ improvement can be observed. In fact, the proposed detector performs close (or marginally better) to the combined ZF-ML detector, however at a much lower complexity as described next.

The complexity of the proposed detector is in terms of average search space size $\beta$ evaluated according to (20) is shown in Fig. 6. Note that unlike in ML detector and combined ZF-ML detector, $\beta$ is SNR dependent for the proposed algorithm. As can be seen from Fig. $6, \beta$ varies from $\beta=3.2$ to 0.2 for the SNR range of 0 to $20 \mathrm{~dB}$. Also, for the ML detector $\beta=16$ and for the combined ZF-ML detector $\beta=4$, irrespective of SNR. For the proposed detector, approximately $\beta=1$ at $\mathrm{SNR}=10 \mathrm{~dB}$. This implies a $75 \%$ reduction in computational complexity compared to the combined ZF-ML detector and a $94 \%$ reduction in computational complexity compared to the ML detector. The computational reduction of the proposed 


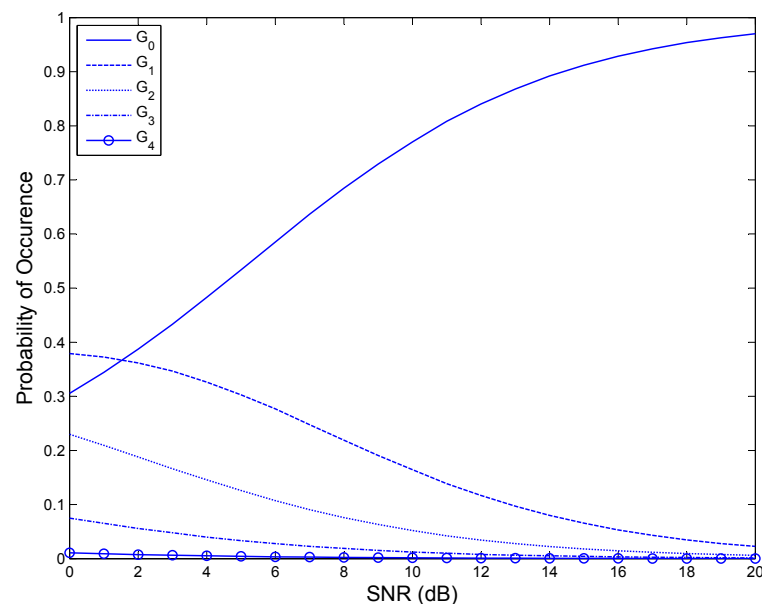

Fig. 4. Probability of occurrence of the classes $P\left(\mathcal{G}_{i}\right)$ versus SNR for the $\mathrm{ZF}$ equalizer.

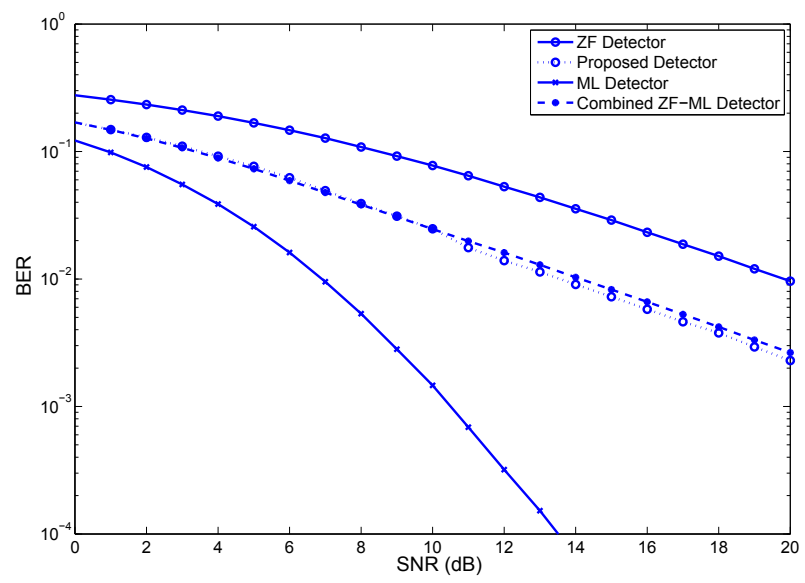

Fig. 5. BER performance versus SNR with the ZF equalizer.

detector is more for high SNR values, i.e. SNR $>10 \mathrm{~dB}$.

Remark 5: We have only presented the results of the proposed detector with ZF based linear equalizer. Simulation results were obtained also with the MMSE based linear equalizer, however omitted due to space limitations. With the proposed detector a BER improvement of $8 \mathrm{~dB}$ was observed compared to the MMSE detector at $\mathrm{BER}=10^{-2}$. This performance is similar to that of the combined MMSE-ML detector [4] but was achieved with approximately $80 \%$ reduction in search space.

\section{CONCLUSIONS}

In this paper, a classifier based low-complexity MIMO detection technique was presented for spacial multiplexing systems. The optimal maximum-likelihood (ML) detector uses a computationally prohibitive search space. The proposed classifier based MIMO detector subdivides the full ML search space into number of variable size subspaces with respect to the solution given by a linear equalizer ( $\mathrm{ZF}$ or MMSE equalizer). Using a decision measure and the Bayesian clas-

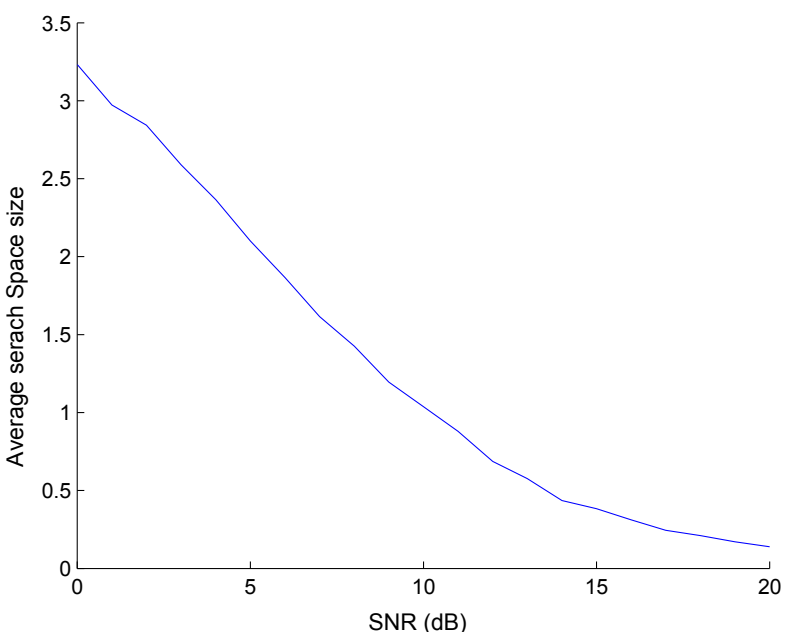

Fig. 6. Average search space size $(\beta)$ versus SNR for the proposed detector with ZF equalizer. For the ML detector $\beta=16$ and for the combined ZF-ML detector $\beta=4$, for all SNRs.

sification concept a specific subspace is selected for each received signal vector. Within the selected subspace a ML type search is performed to obtain the final detected vector. The computational advantage of the proposed technique results from the two reasons: (a) Only a single subspace is selected instead of the full ML space for transmitted symbol vector. (b) Subspaces with larger size occurs with lower probability and vise versa. The average search space size for the proposed detector is SNR dependent and decreases monotonically as the SNR increases. At $\mathrm{SNR}=10 \mathrm{~dB}$, an average search space reduction of approximately $75 \%$ can be achieved with the proposed detector compared to the combined ZF-ML detector with the same BER performance.

For higher dimensional MIMO systems and larger symbol constellations proper selection of error classes is essential to obtain the best performance versus complexity trade-off with the proposed detector.

\section{ACKNOWLEDGMENT}

This work was supported by the Australian Research Council (ARC) Discovery Project Number 0558865.

\section{REFERENCES}

[1] H. Artes, D. Seethaler, and F. Hlawatsch, "Efficient detection algorithms for MIMO channels: A geometrical approach to approximate ML detection”, IEEE Transactions on Signal Processing, Vol. 51, No. 11, pp. 2808-2820, November 2003.

[2] E. Viterbo and J. Boutros, "A universal lattice code decoder for fading channels", IEEE Transactions on Information Theory, Vol. 45, No. 5, pp. 1639-1642, July 1999.

[3] V. Pammer, Y. Delignon, W. Sawaya, and D. Boulinguez, "A low complexity suboptimal MIMO receiver: The combined ZF-MLD algorithm", IEEE PIMRC'03, Vol 3, pp. 2271-2275, September 2003.

[4] L. Rugini, P. Banelli, and G. B. Giannakis, "MMSE-based local ML detection of linearly precoded OFDM signals", IEEE ICC'04, Vol. 6, pp. 3270-3275, June 2004.

[5] J. G. Proakis, Digital Communications, McGraw-Hill Series in Electrical and Computer Engineering, 4th Edition, New York:McGraw-Hill, 2001. 